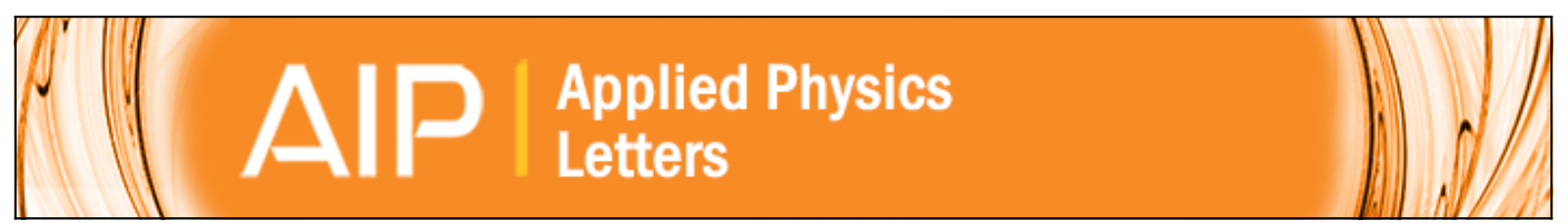

\title{
Suppression of cross-hatched polariton disorder in GaAs/AIAs microcavities by strain compensation
}

Joanna M. Zajac, Edmund Clarke, and Wolfgang Langbein

Citation: Applied Physics Letters 101, 041114 (2012); doi: 10.1063/1.4739245

View online: http://dx.doi.org/10.1063/1.4739245

View Table of Contents: http://scitation.aip.org/content/aip/journal/apl/101/4?ver=pdfcov

Published by the AIP Publishing

\section{Articles you may be interested in}

Polariton condensation in a strain-compensated planar microcavity with InGaAs quantum wells

Appl. Phys. Lett. 105, 191118 (2014); 10.1063/1.4901814

GaN hybrid microcavities in the strong coupling regime grown by metal-organic chemical vapor deposition on sapphire substrates

J. Appl. Phys. 101, 093110 (2007); 10.1063/1.2728744

Room temperature polariton luminescence from a Ga N/AI Ga N quantum well microcavity

Appl. Phys. Lett. 89, 071107 (2006); 10.1063/1.2335404

Fabrication of InAs quantum dots in Al As/Ga As DBR pillar microcavities for single photon sources

J. Appl. Phys. 97, 073507 (2005); 10.1063/1.1882764

An angle modulation reflectance spectroscopy characterization of a GaAs/GaAlAs asymmetric microcavity structure

Appl. Phys. Lett. 84, 194 (2004); 10.1063/1.1639938

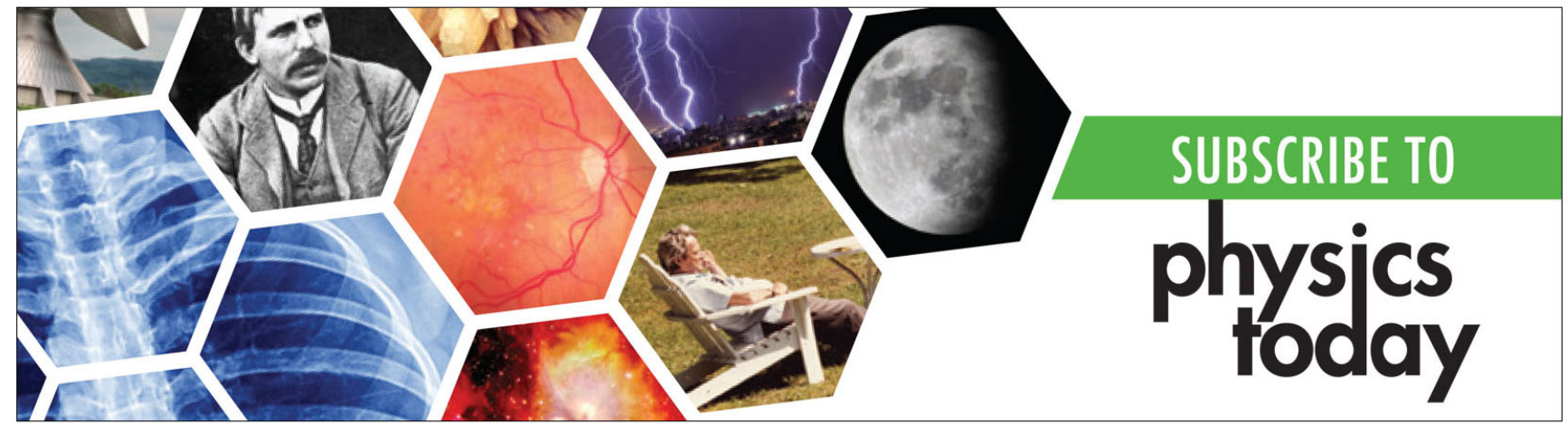




\title{
Suppression of cross-hatched polariton disorder in GaAs/AIAs microcavities by strain compensation
}

\author{
Joanna M. Zajac, ${ }^{1, a)}$ Edmund Clarke, ${ }^{2}$ and Wolfgang Langbein ${ }^{1}$ \\ ${ }^{1}$ School of Physics and Astronomy, Cardiff University, The Parade, Cardiff CF24 3AA, United Kingdom \\ ${ }^{2}$ Department of Electronic and Electrical Engineering, University of Sheffield, Mappin Street, \\ Sheffield S1 4JD, United Kingdom
}

(Received 4 June 2012; accepted 11 July 2012; published online 25 July 2012)

\begin{abstract}
Zinc-blende semiconductor heterostructures grown in the [001] direction with a small lattice mismatch accommodate stress by developing a cross-hatch dislocation pattern. In GaAs based planar microcavities grown by molecular beam epitaxy, this pattern creates a potential landscape for exciton-polaritons, causing scattering and localization. We report here on suppressing the cross-hatch by introducing strain-compensating AlP layers into the center of the low index AlAs layers of the distributed Bragg reflectors. We observe a reduction of the cross-hatch dislocation density by at least one order of magnitude for $1.1 \mathrm{~nm}$ thick AlP layers, which correspond to an effective $\mathrm{AlAs}_{0.985} \mathrm{P}_{0.015}$ low index layer. These compensated structures show a remaining polariton disorder potential in the $10 \mu \mathrm{eV}$ range. (C) 2012 American Institute of Physics.
\end{abstract}

[http://dx.doi.org/10.1063/1.4739245]

The lattice constants of GaAs and AlAs layers differ by $0.14 \%$ at room temperature, leading to strain in AlAs layers grown on GaAs substrates, which for a film thickness above a critical thickness ${ }^{1}$ relaxes by the formation of a cross-hatch dislocation pattern. In GaAs/AlAs microcavities such crosshatches influence the propagation of polaritons and give rise to elastic scattering and the appearance of a cross-shaped signature in the far-field emission from a microcavity. ${ }^{2-6}$ Furthermore, they can cause a localization of polariton condensates observed in such microcavities in the strong coupling regime. ${ }^{7}$ In order to observe polariton fluid propagation over distances exceeding hundreds of $\mu \mathrm{m}$, and spontaneous vortex dynamics in a homogeneous polariton superfluid, the cross-hatch pattern needs to be suppressed. One possibility to reduce the strain and thus avoid misfit dislocation formation is p or n-type doping with small atoms, ${ }^{8}$ such as carbon or silicon, respectively. This can be used in electrically pumped structures such as vertical cavity surface emitting lasers (VCSELs). However, the doping gives rise to free-carrier absorption, limiting the lifetime and thus the propagation length of polaritons. Moreover, the doping introduces electric fields which cause energy shifts of the quantum well (QW) excitons due to the Stark effect, and free carriers which broaden the QW exciton due to carrier-carrier scattering.

In the present work, we propose and demonstrate the suppression of the cross-hatch dislocation pattern by the incorporation of strain compensating AlP layers into the AlAs layers of the distributed Bragg reflectors (DBRs).

Thin films of cubic semiconductors grown on (001) substrates with a moderate lattice mismatch $\varepsilon_{\mathrm{m}}<2 \%$ grow in a layer-by-layer mode. For layer thicknesses exceeding the critical thickness, ${ }^{1}$ the strain relaxes by the formation of misfit dislocations arrays. A misfit dislocation (MD) is a mixed edge and screw dislocation, ${ }^{9}$ as shown in Fig. 1(a) where the

${ }^{\text {a)} E l e c t r o n i c ~ m a i l: ~ Z a j a c J M @ c a r d i f f . a c . u k . ~}$
Burgers vector $\mathbf{b}_{\mathrm{MD}}$ of the MD is decomposed into an edge dislocation ( $\left.\mathbf{b}_{\text {edge }}\right)$ and a screw dislocation $\left(\mathbf{b}_{\text {screw }}\right)$. The MD line lies in the heterointerface plane, while the accompanying threading dislocation (TD) line lies in a plane perpendicular to the heterointerface. TDs either propagate from the substrate or are created in the epilayers. In GaAs/AlAs multilayers, the TDs propagation from the substrate is suppressed ${ }^{10}$ by a deflection into the interface plane along the $\langle 110\rangle$ directions.

The mechanism behind the formation of the hatches parallel to the [110] and [110] directions was identified in Ref. 1 for $\mathrm{GaAs} / \mathrm{GaAs}_{0.5} \mathrm{P}_{0.5}$ heterostructures grown on a (100) GaAs substrate with $\varepsilon_{\mathrm{m}}=1.8 \%$. Using TEM and SEM, bending of threading dislocations on the heterointerfaces on $\{111\}$ slip planes was observed, providing a mechanism for the formation of MD arrays sketched in Fig. 1(b). Following the notation given in Fig. 1(a), the critical thickness is calculated as ${ }^{1}$

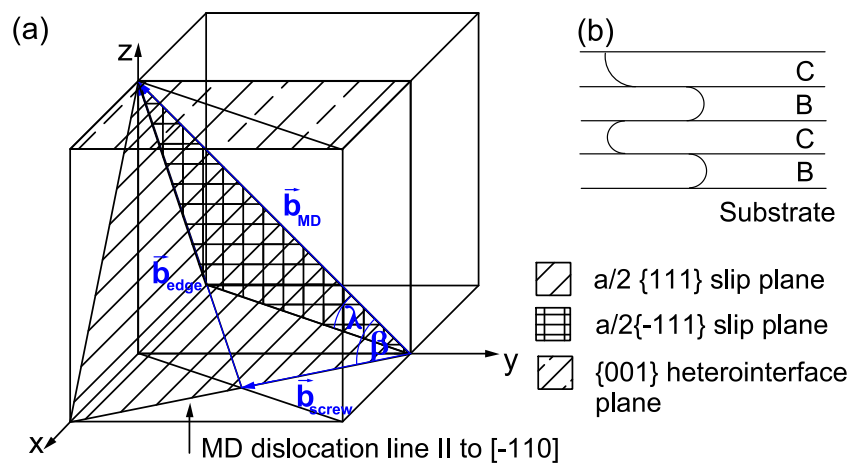

FIG. 1. (a) Sketch of the MD development in GaAs/AlAs heterostructures. The angle between the dislocation line and its Burgers vector is $\beta=60^{\circ}$, and the angle between the Burgers vector and a line in the plane of the heterointerface perpendicular to the dislocation line is $\lambda=60^{\circ}$. (b) Sketch of the dislocation array development on the (111) slip plane, B and C denote different layers. 


$$
h_{\mathrm{c}}=\frac{b}{4 \pi \varepsilon_{\mathrm{m}}}\left(1+\ln \frac{h_{\mathrm{c}}}{b}\right) \frac{1-\nu \cos ^{2}(\beta)}{(1+\nu) \cos (\lambda)},
$$

where $b=|\mathbf{b}|, \quad \varepsilon_{\mathrm{m}}=\left(a_{\mathrm{s}}-a_{\mathrm{f}}\right) / a_{\mathrm{f}}$ is the misfit strain where $a_{\mathrm{s}}$ and $a_{\mathrm{f}}$ are the lattice constants of the substrate and the film, respectively, $\nu=C_{12} /\left(C_{11}+C_{12}\right)$ is the Poisson ratio of the film, $\alpha$ is the angle between the dislocation line and $\mathbf{b}$, and $\lambda$ is the angle between $\mathbf{b}$ and the direction in the film plane perpendicular to the intersection of the slip plane with the interface.

Due to the different thermal expansion coefficients $\alpha$ of GaAs and AlAs (see Table I), they are expected to be lattice matched ${ }^{13}$ around $900{ }^{\circ} \mathrm{C}$. The critical thickness for the growth of AlAs on a GaAs substrate at a temperature of $590{ }^{\circ} \mathrm{C}$ calculated using Eq. (1) is $h_{\mathrm{c}} \sim 0.6 \mu \mathrm{m}$, with $b=a / \sqrt{2}$ as shown in Fig. 1(a). For a full GaAs/AlAs Bragg period instead, using its average lattice constant, we find $h_{\mathrm{c}} \approx 1.1 \mu \mathrm{m}$.

Strain relaxation occurs gradually with increasing thickness $h>h_{\text {c }}$, creating an equilibrium MD density ${ }^{9}$

$$
\rho_{\mathrm{MD}}=\frac{\varepsilon_{\mathrm{m}}}{b_{\|}}\left(1-\frac{h_{\mathrm{c}}}{h}\right)=\frac{\varepsilon_{\mathrm{m}}}{b_{\|}} R,
$$

where $b_{\|}=b \cos (\lambda)$ and $R$ being the fractional strain relaxation. For a AlAs/GaAs Bragg mirror of $h=3.5 \mu \mathrm{m}$, we find $\rho_{\mathrm{MD}}=2 / \mu \mathrm{m}$ corresponding to $R=68 \%$. We measured a cross hatch density of $\rho_{\mathrm{MD}} \sim 30 / \mathrm{mm}$, corresponding to $R=1.5 \%$. The MD formation thus remained far from equilibrium during the growth.

However, even this small MD density is affecting the polariton propagation in the MC. In order to reduce the MD density one needs to reduce the lattice mismatch of the DBR. To do this, we sandwich a thin AlP layer into the center of each AlAs layer of the DBR to obtain an effective lattice matched $\mathrm{AlAs}_{1-x} \mathrm{P}_{x}$ ternary alloy as low index layer. Growing a thin AlP layer instead of an alloy provides a better control over the composition, specifically for the group $\mathrm{V}$ alloys. Additionally, we avoid interfaces in which elements of both groups III and V change, which can lead to the interface layers modifying the band offset. Furthermore, by adding the lower index material AlP in the center of the low index layer, we enhance its reflectivity.

The values of the phosphorus fraction $x$ used in our study were chosen to lattice-match $\mathrm{AlAs}_{1-x} \mathrm{P}_{x}$ to GaAs at

TABLE I. GaAs, AlAs, and AlP lattice constants $a$, thermal expansion coefficients $\alpha$, and refractive indices $n$ at $850 \mathrm{~nm}$ wavelength at room temperature.

\begin{tabular}{lccc}
\hline \hline Parameter & GaAs & AlAs & AlP \\
\hline$A(\mathrm{~nm})$ & $0.56533^{\mathrm{a}}$ & $0.566162^{\mathrm{a}}$ & $0.54635^{\mathrm{b}}$ \\
$\alpha\left(10^{-6} / \mathrm{K}\right)$ & $6.24,^{\mathrm{a}} 6.86^{\mathrm{a}}$ & $5.2^{\mathrm{c}}$ & $4.5^{\mathrm{d}}$ \\
$\mathrm{N}$ & $3.567^{\mathrm{e}}$ & $2.97^{\mathrm{e}}$ & $2.775^{\mathrm{f}}$ \\
\hline \hline
\end{tabular}

${ }^{\mathrm{a}}$ Reference 11.

${ }^{\mathrm{b}}$ Reference 12 .

${ }^{\mathrm{c}}$ Reference 13 .

${ }^{\mathrm{d}}$ Reference 14.

e Reference 15.

${ }^{\mathrm{f}}$ Reference 16.
TABLE II. Parameters of the investigated microcavity samples.

\begin{tabular}{lccccc}
\hline \hline P content in AlAs ${ }_{1-x} \mathrm{P}_{x}(\%)$ & 0 & 1 & 1.5 & 2 & 3 \\
\hline AlP layer thickness $(\mathrm{nm})$ & 0 & 0.8 & 1.1 & 1.5 & 2.2 \\
Matching temperature $\left({ }^{\circ} \mathrm{C}\right)$ & 1100 & 830 & 700 & 560 & 300 \\
Cavity mode wavelength $\lambda_{\mathrm{c}}(\mathrm{nm})$ & 866 & 873 & 851 & 856 & 855 \\
\hline \hline
\end{tabular}

different temperatures as given in Table II. The temperature dependent lattice constant $a(T)$ was calculated using the linear thermal expansion

$$
a(T)=a\left(T_{0}\right)\left(1+\alpha\left(T-T_{0}\right)\right)
$$

where $\alpha$ is the thermal expansion coefficient. Using $\alpha_{\mathrm{GaAs}}$ from Ref. 11, GaAs and AlAs are lattice matched at $T=1400^{\circ} \mathrm{C}$, while for $\alpha_{\mathrm{GaAs}}$ from Ref. 13 they are matched at $T=900^{\circ} \mathrm{C}$. In the sample design, we used the average of both values. The lattice constant of the ternary alloy was calculated using the Vegard's law from the lattice constants of the binary alloys and thermal expansion coefficients as listed in Table I. Five microcavity samples with nominal phosphorus contents of $x=0$ to $3 \%$ were grown (see Table II). They consist of a bare $2 \lambda_{\mathrm{c}}$ GaAs cavity layer surrounded by $\mathrm{AlAs}_{1-x} \mathrm{P}_{x} / \mathrm{GaAs}$ DBRs with 26 and $23 \lambda_{\mathrm{c}} / 4$ pairs on the bottom and top, respectively. The calculated temperature dependence of the lattice mismatch $\varepsilon_{\mathrm{m}}$ of $\mathrm{AlAs}_{1-x} \mathrm{P}_{x}$ corresponding to the different samples is shown in Fig. 2(a).

Resonant transmission was measured in order to determine the average density of cross-hatches. The samples were held in a bath cryostat at a temperature of $T=80 \mathrm{~K}$ to avoid GaAs absorption at the cavity resonance. The samples were illuminated from the substrate side and imaged from the epi side using 0.5NA lenses. A detailed description of the setup can be found in Ref. 17. The measured cavity mode wavelengths are shown in Table $\mathrm{I}$ and are red detuned by $50-80 \mathrm{meV}$ from the bulk GaAs exciton ${ }^{18}$ of the cavity layer at $1.508 \mathrm{eV}$. The real space transmission images of the samples under illumination with a nearly collimated beam of resonant $150 \mathrm{fs}$ pulses and $250 \mu \mathrm{m}$ diameter are given in Fig. 2(b). The observed cross-hatch density shows a clear dependence on the strain compensation. The resulting measured cross-hatch density versus the thickness of the AlP layer (see Fig. 2(c)) shows a minimum at $1.1 \mathrm{~nm}$ corresponding to a low index layer of $\mathrm{AlAs}_{0.985} \mathrm{P}_{0.015}$, which is nominally lattice matched at $700{ }^{\circ} \mathrm{C}$, close to the growth temperature of $590^{\circ} \mathrm{C}$. Furthermore, the density reduction has a wider range for the $[1 \overline{1} 0]$ compared to the $[110]$ direction. The non-equivalence of the two directions is related to the breaking of the inversion symmetry by the lattice basis and is not observed in $\mathrm{Si} / \mathrm{SiGe}$ structures. ${ }^{19}$ The varying density of point-like defects seen in the images - specifically the high density in the $\mathrm{AlAs}_{0.985} \mathrm{P}_{0.015}$ sample is attributed to a faulty lip heater in the Ga Veeco "SUMO" cell of the VG V90 MBE (molecular beam epitaxy) machine. The influence of such defects on the polariton states are characterized in Ref. 17.

To exemplify the effect of the suppressed cross-hatch disorder on the polariton states, we compare in Fig. 3 the polariton propagation in $0 \%$ and $1.5 \%$ samples. Without 
(a)

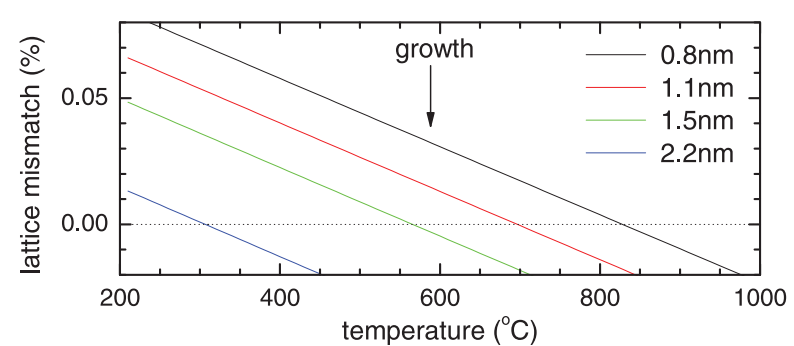

(b)

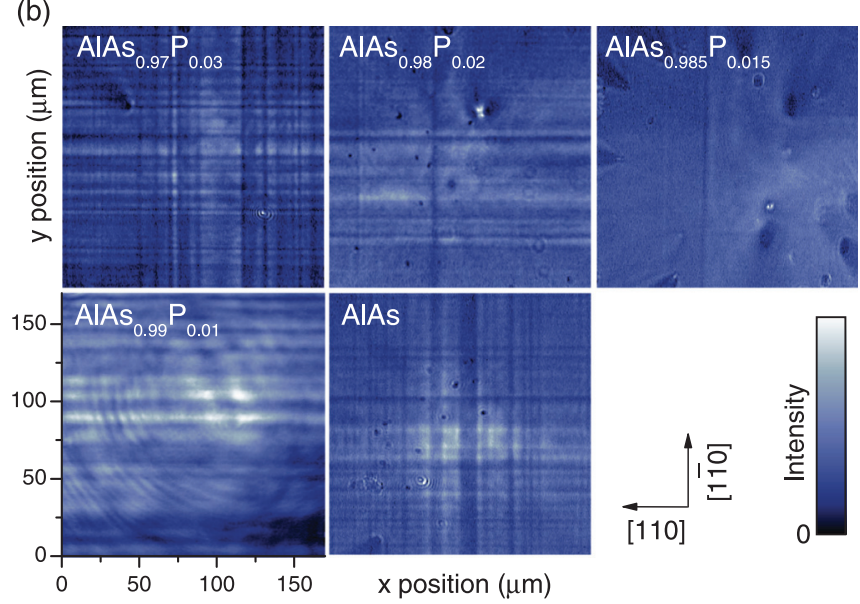

(c)

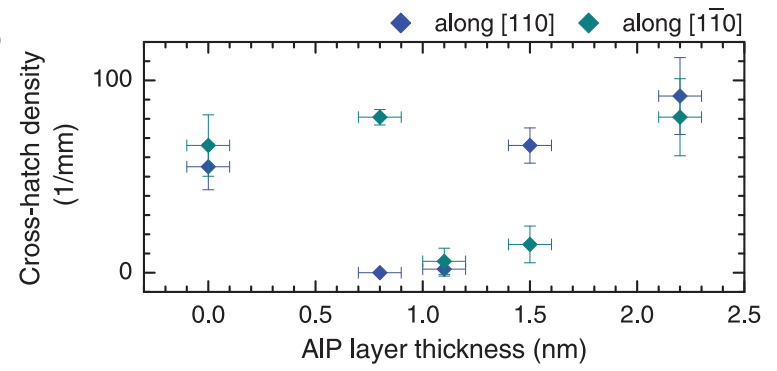

FIG. 2. (a) Calculated temperature dependence of the lattice mismatch $\varepsilon_{\mathrm{m}}$ of $\mathrm{AlAs}_{1-x} \mathrm{P}_{x}$ for different AlP layer thicknesses as indicated. Parameters are taken from Table I. (b) Real space transmission images of the investigated microcavities with different strain compensation. Intensity on a linear color scale as indicated. (c) cross-hatch density as function of AlP layer thickness. Error bars for the layer thickness are estimated from the shutter transit times.

disorder, they exhibit a in-plane quadratic dispersion with a minimum at the band edge $\hbar \omega_{\mathrm{c}}$ at $1.4330(1.4565) \mathrm{eV}$ for the $0 \%(1.5 \%)$ sample, respectively, and an effective mass of $m=2 \times 10^{-5} m_{\mathrm{e}}$ determined from the measured dispersion, where $m_{\mathrm{e}}$ is the free electron mass. We use a focused excitation with a diffraction limited spot of about $25 \mu \mathrm{m}$ size, containing wavevectors $|\mathbf{k}|<0.3 / \mu \mathrm{m}$. Exciting with a cw-laser at the polariton band-edge, we select the polariton states close to the zero momentum. The excited polariton states (Fig. 3(a)) are strongly affected by the cross-hatch disorder, being localized between hatches. In the $1.5 \%$ sample instead, the virtual absence of the cross-hatch disorder enables the nearly free propagation of the excited polaritons. The interference fringes created by the scattering from the defects show a period of about $20 \mu \mathrm{m}$, corresponding to $|\mathbf{k}|=0.16 / \mu \mathrm{m}$, and a kinetic energy of $47 \mu \mathrm{eV}$. The free propagation at such a low kinetic energy gives an upper limit of about $20 \mu \mathrm{eV}$ for the residual disorder. The single horizontal MD hatch present in the investigated region leads to the reduction of the emission in the hatch, evidence for an attrac-
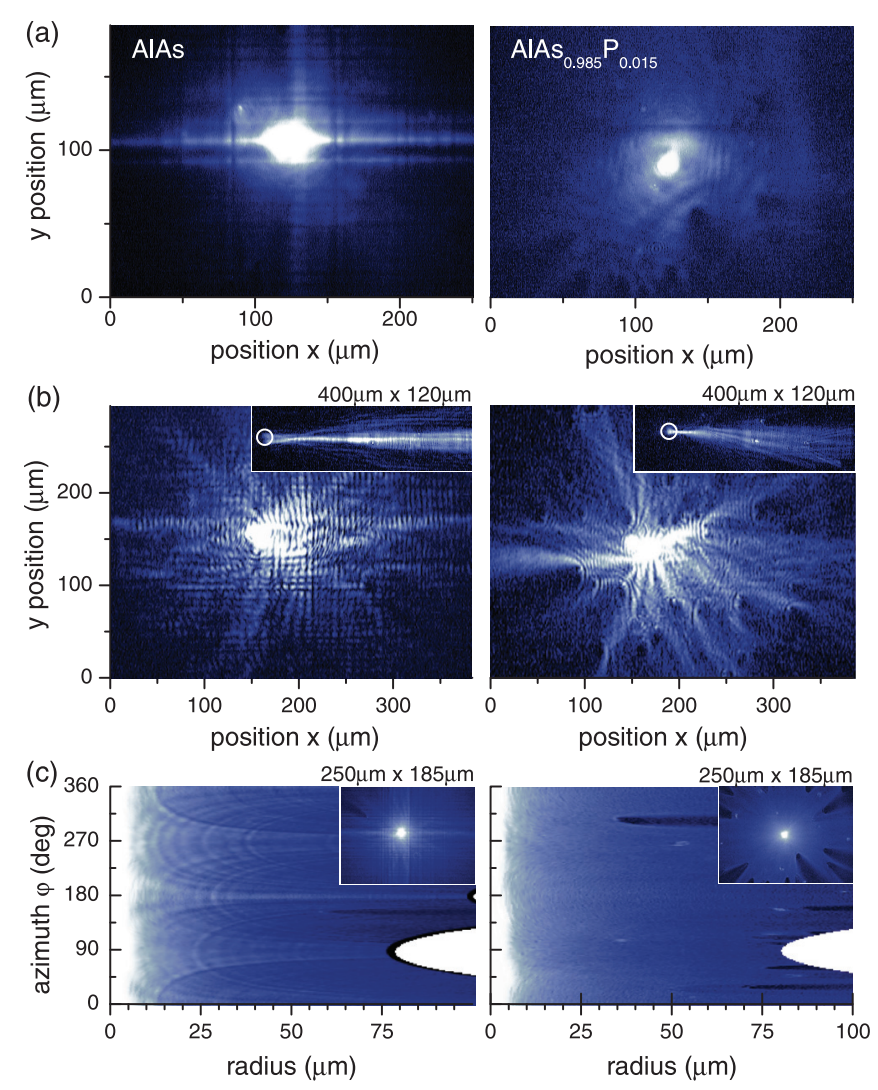

FIG. 3. Real space transmission images of the samples with no strain compensation (left column) and optimum strain compensation (right column). Color scale as in Fig. 2. Excitation energy relative to band edge were $0 \mathrm{meV}$ in (a), $1 \mathrm{meV}$ in (b), $6 \mathrm{meV}$ in the inset of (b), and $0-10 \mathrm{meV}$ in (c). Excitation wavevectors were $|\mathbf{k}|<0.3 / \mu \mathrm{m}$ in (a), $|\mathbf{k}-(1.1,0) / \mu \mathrm{m}|<0.3 / \mu \mathrm{m}$ in (b), $|\mathbf{k}-(2.3,0) / \mu \mathrm{m}|<0.3 / \mu \mathrm{m}$ in the inset of (b), and $|\mathbf{k}|<4 / \mu \mathrm{m}$ in (c).

tive potential increasing the local wavevector and the group velocity and thus reducing the polariton density in the hatch. Tuning the excitation energy about $1 \mathrm{meV}$ above the polariton bandgap, polaritons propagate over distances in the order of $200 \mu \mathrm{m}$ as seen in Fig. 3(b). Polaritons excited with even higher kinetic energies of $6 \mathrm{meV}$ and a wavevector $\mathbf{k}$ centered at $(2.3,0) / \mu \mathrm{m}$ (see insets of Fig. 3(b), the excitation position is indicated as a circle) propagate over hundreds of micrometers in the $x$ direction. Fitting the data we deduced exponential decay lengths in the order of $1 \mathrm{~mm}$. In the $0 \%$ sample, the propagation proceeds along the waveguide channels ${ }^{5}$ provided by the cross-hatches, while in the $1.5 \%$ sample, the excited wavepacket is spreading according to its free propagation. Exciting instead with $150 \mathrm{fs}$ pulses covering kinetic energies from zero to $10 \mathrm{meV}$ and focusing in real space to a diffraction limited spot of $1.5 \mu \mathrm{m}$ size covering $|\mathbf{k}|<4 / \mu \mathrm{m}$, polaritons with a large range of kinetic energies are propagating away from the excitation spot (see inset of Fig. 3(c)). The interference pattern is suppressed by the short coherence length of the excited polariton wavepacket. To observe the omnidirectional propagation, we show the data in cylindrical coordinates centred at the excitation spot in Fig. 3(c). In the $0 \%$ sample the propagation is anisotropic with the longest propagation length along the hatch directions [110] and [110]. In the $1.5 \%$ sample instead, an isotropic propagation is observed, apart from the point-like defects. 
In conclusion, we have proposed and demonstrated the suppression of the cross-hatch disorder in GaAs/AlAs microcavities by strain compensation, which was realized by incorporating thin AIP layers into the AlAs layers of the DBRs. Using an AlP layer thickness of $1.1 \mathrm{~nm}$, corresponding to an effective $\mathrm{AlAs}_{0.985} \mathrm{P}_{0.015}$ alloy of the low-index layer, a reduction of the cross hatch density by an order of magnitude is achieved, which reduces the residual polariton disorder potential to the $10 \mu \mathrm{eV}$ range. This progress is important for the study of intrinsic collective polariton phenomena such as superfluidity, vortices and solitons.

This work was supported by the EPSRC UK Research Council (Grant No. EP/F027958/1). We acknowledge discussions with Pavlos Lagoudakis. The investigated structures were grown by E.C. at the EPSRC National Centre for III-V Technologies, Sheffield, UK.

${ }^{1}$ J. W. Matthews and A. E. Blakeslee, J. Cryst. Growth 27, 118 (1974).

${ }^{2}$ M. Gurioli, F. Bogani, D. S. Wiersma, P. Roussignol, G. Cassabois, G. Khitrova, and H. Gibbs, Phys. Rev. B 64, 165309 (2001).

${ }^{3}$ W. Langbein and J. M. Hvam, Phys. Rev. Lett. 88, 047401 (2002).

${ }^{4}$ W. Langbein, in Proceedings of 26th International Conference on Physics of Semiconductors, Edinburgh, UK, 2002.

${ }^{5}$ W. Langbein, Rivista del Nuovo Cimento 33, 255 (2010).
${ }^{6}$ M. Abbarchi, C. Diederichs, L. Largeau, V. Ardizzone, O. Mauguin, T. Lecomte, A. Lemaitre, J. Bloch, P. Roussignol, and J. Tignon, Phys. Rev. B 85, 045316 (2012).

${ }^{7}$ J. Kasprzak, M. Richard, S. Kundermann, A. Baas, P. Jeambrun, J. M. J. Keeling, F. M. Marchetti, M. H. Szymanska, R. Andre, J. L. Staehli, V. Savona, P. B. Littlewood, B. Deveaud, and L. S. Dang, Nature (London) 443, 409 (2006).

${ }^{8}$ A. Mazuelas, R. Hey, B. Jenichen, and H. T. Grahn, Appl. Phys. Lett. 70, 2088 (1997).

${ }^{9}$ A. M. Andrews, J. S. Speck, A. E. Romanov, M. Bobeth, and W. Pompe, J. Appl. Phys. 91, 1933 (2002).

${ }^{10}$ M. Shinohara, T. Ito, and Y. Imamura, J. Appl. Phys 58, 3449 (1985).

${ }^{11}$ EMIS Data Review Series No 7, Properties of Aluminium Galium Arsenide, edited by B. L. Weiss (INSPEC, 1993).

${ }^{12}$ Y. Singh, Semiconductor Devices (I.K. International Publishing House, 2009).

${ }^{13}$ M. Ettenberg and R. Paff, J. Appl. Phys. 41, 3926 (1970).

${ }^{14}$ Landolt-Bornstein Numerical data and functional relationships in science and technology, Vol 17, Semiconductors, Physics of group IV elements and III-V compounds, edited by H. W. O. Madelung and M. Schulz (Springer, Berlin, 1982).

${ }^{15}$ S. Gehrsitz, F. K. Reinhart, C. Gourgon, N. Herres, A. Vonlanthen, and H. Sigg, J. Appl. Phys. 87, 7825 (2000).

${ }^{16}$ B. Monemar, Solid State Commun. 8, 1295 (1970).

${ }^{17}$ J. M. Zajac, W. Langbein, M. Hugues, and M. Hopkinson, Phys. Rev. B 85, 165309 (2012).

${ }^{18}$ Y. Chen, A. Tredicucci, and F. Bassani, Phys. Rev. B 52, 1800 (1995).

${ }^{19}$ S. Y. Shiryaev, F. Jensen, J. L. Hansen, J. W. Petersen, and A. N. Larsen, Phys. Rev. Lett. 78, 503 (1997). 